\title{
On the Line Congruences
}

\author{
Ferhat Taş* and Osman Gürsoy
}

(Communicated by Levent Kula)

\begin{abstract}
The purpose of this paper is to find the quantities and surfaces of a line congruence via examining it in the dual space and to represent the results more appropriately for computational approximations. For this, we take mainly two-dual parameter motion on the dual unit sphere (DUS) so, we get a line congruence corresponding this motion by a new method. Thus, the equations of the developable surfaces, the principal surfaces, the focal surfaces and the center surface of the line congruence are found by coordinate functions. The results are illustrated by examples.
\end{abstract}

Keywords: Differential line geometry; Ruled surface; Developable ruled surface.

AMS Subject Classification (2010): Primary: 51M30; Secondary: 53A25.

\section{Introduction}

Line geometry generally investigates the lines of 3-dimensional real space and their motions. These lines construct a line space. Projective, dual and vectorial representations are used to examine the properties of elements of this space. Plücker [18] is the first person who represented the lines by ordered six-tuples. The first three elements of this ordered six-tuples represent the coordinates of direction vector and the last three elements represent the moment vector of this direction vector with respect to the origin of a line. Study [22], one of the Plücker' s students, moved this representation of lines to the dual vector space via combining dual numbers which constructed by Clifford [5]. Thus, he showed that a line corresponds to a point on the DUS.

Line complexes, line congruences and ruled surfaces are elements of the line space. These elements are described by a three-parameter, two-parameter and one-parameter motion of a line in 3-dimensional real space, respectively and are often used in optic, mechanic, space kinematics and robot motion planning. That is why computation of differential geometric properties of these elements are important.

In Euclidean 3-space, a two-parameter set of lines is called a line congruence. For instance, the normal vector

field of a surface constitute such a line congruence. So it is in the form of a director or reference surface and a unit direction vector field. There are a lot of study about line congruences. Previously, this subject was a theoretical, especially in the application of optics began to gain importance. The importance of line congruences has also increased with the developing technology. In today's technology, the motion of a hot wire at the end of a robotic arm is essentially the movement of a line in space.

Blasckhe [3], investigated the line congruences to found its geometric properties and certain surfaces in IDModule. Shephard [21], studied about first order invariants of line congruences using the method of moving frames and gave a geometric meaning of these invariants. Larochelle and tec. [16], aimed to combine $4 \mathrm{C}$ mechanism with a line congruence for visualization. For this process, they firstly determine the representation of line congruences which appropriate for both workstation and immersive virtual reality computer graphics. Jüttler and Rittenschober [14], studied about determination of rational curves and surfaces on the line congruence. Abdel-Baky [1], gave differential geometric properties to classify the line congruences. Odehnal [17], found a way to the rational parametrization of isotropic line congruences.

For example, Köse [15] revealed a method for determination of developable ruled surfaces of a line congruences. But his method's solution belongs to the special parameters. His determination is not general for describing the developable surfaces of a line congruence. 
In this paper, we found general equations to determine the surfaces of a line congruence. For the convenience of some long term equations, abbreviations have been used for trigonometric functions such as: $\cos (u):=$ $c(u), \sin (u):=s(u)$

\section{Fundamentals}

\subsection{Line geometry}

In this section, we introduce some elements of the line space.

2.1.1. Ruled surface. Ruled surfaces are the popular topic in geometry and mechanisms. A ruled surface can be given in point coordinate form as

$$
\mathbf{r}(u, v)=\mathbf{a}(u)+\lambda \mathbf{x}(u),
$$

where $\lambda \in \mathbb{R}, \mathbf{a}, \mathbf{x} \in \mathbb{R}^{3}$. $\mathbf{a}(u)$ and $\mathbf{x}(u)$ are base curve and directrix, respectively. There is two type of ruled surfaces: developable and non-developable (skew). Cylinder and cone are example of the developable ruled surface while hyperboloid and helicoid are examples of skew ruled surfaces. In line geometry, from a kinematic point of view, a one-parameter motion of a line $L$ in 3-dimensional real space represents a ruled surface: $L(u)$, $[3,8,9,10,19]$.

2.1.2. Line congruence. In $\mathbb{R}^{3}$, a two-parameter family of lines can be defined as

$$
\mathbf{k}(u, v, w)=\mathbf{a}(u, v)+w \mathbf{x}(u, v)
$$

where $w \in \mathbb{R}, \mathbf{a}, \mathbf{x}: D \subset \mathbb{R}^{2} \rightarrow \mathbb{R}^{3}, \mathbf{k}: D \times \mathbb{R} \rightarrow \mathbb{R}^{3}, \mathbf{a}(u, v)$ and $\mathbf{x}(u, v)$ are base surface and generator vector field, respectively. Thus, $\mathbf{k}$ draw a line congruence in 3-dimensional real space. For example, the tangent vector field of a regular surface constitute a line congruence. Line congruences are geometric objects that include many types of ruled surfaces, $[3,8,19]$.

Another representation of the elements of the line space will be given in the next section.

\subsection{Study model}

A dual number is represented by $a+\varepsilon b, a, b \in \mathbb{R}$ where $\varepsilon^{2}=0$. Here we use symbol $\mathbb{D}$ for the set of dual numbers. The number $1+\varepsilon .0$ is called dual unit number and represented by ordered pair $(1,0)$. If $f$ is an analytic function then it can be expanded to the power series of the function which is converging in a certain interval: $f(x)=\sum_{k=0}^{\infty} a_{k}\left(x-x_{0}\right)^{k}$. Then, if we apply this definition to the dual parameter function we get

$$
f(x+\varepsilon y)=\sum_{k=0}^{\infty} a_{k}\left(x+\varepsilon y-x_{0}\right)^{k}=\sum_{k=0}^{\infty} a_{k}\left(x-x_{0}\right)^{k}+\varepsilon y \sum_{k=0}^{\infty} k a_{k}\left(x-x_{0}\right)^{k-1}=f(x)+\varepsilon y f^{\prime}(x) .
$$

We can give some examples to concretize, $\sin (x+\varepsilon y)=\sin (x)+\varepsilon y \cos (x), \cos (x+\varepsilon y)=\cos (x)-$ $\varepsilon y \sin (x), \exp (x+\varepsilon y)=\exp (x)+\varepsilon y \exp (x)$.

The set $\mathbb{D}^{3}=\mathbb{D} \times \mathbb{D} \times \mathbb{D}$ is a 3-dimensional dual space. The elements of this space are $\mathbf{X}=(\mathbf{x}, \overline{\mathbf{x}})=\mathbf{x}+\varepsilon \overline{\mathbf{x}}$, where $\mathbf{x}, \overline{\mathbf{x}} \in \mathbb{E}^{3}$. $\mathbf{X}$ is called a dual vector.

In $\mathbb{D}^{3}$, the inner product of two dual vectors is defined by

$$
<\mathbf{X}, \mathbf{Y}>=<\mathbf{x}, \mathbf{y}>+\varepsilon(<\mathbf{x}, \overline{\mathbf{y}}>+<\overline{\mathbf{x}}, \mathbf{y}>)
$$

where $\mathbf{X}=\mathbf{x}+\varepsilon \overline{\mathbf{x}}$ and $\mathbf{Y}=\mathbf{y}+\varepsilon \overline{\mathbf{y}}$. Then we can define the dual unit vector: $\|\mathbf{X}\|^{2}=<\mathbf{X}, \mathbf{X}>=\|\mathbf{x}\|^{2}+2 \varepsilon<$ $\mathbf{x}, \overline{\mathbf{x}}>=1$. So, $\mathbf{X}$ is a dual unit vector iff

$$
\|\mathbf{x}\|=1,<\mathbf{x}, \overline{\mathbf{x}}>=0 .
$$

The set of dual unit vectors is called DUS. Therefore, a straight line can be represented by a dual unit vector $\mathbf{X}=\mathbf{x}+\varepsilon \overline{\mathbf{x}}$ in 3-dimensional dual space $\left(\mathbb{D}^{3}\right)$.

As known, a straight line $L$ can be determined by a point $A$ and a unit vector $\mathbf{x}$ in 3-dimensional real space. Let $\overline{\mathbf{x}}$ be moment vector of $L$ with respect to the origin then the normalized Plücker coordinate of a line can be represented by an ordered pair $(\mathbf{x}, \overline{\mathbf{x}})=(\mathbf{x}, \mathbf{a} \times \mathbf{x})$. On the other hand, since the determination of the moment vector is independent of the choice of point on the line we can give the relation: $\mathbf{a}=\mathbf{x} \times \overline{\mathbf{x}}$ $[3,4,6,8,10,15,18,19,20,22,23]$. 
Definition 2.1. The dual angle between two dual vectors $\mathbf{X}$ and $\mathbf{Y}$ can be represented by

$$
\hat{\varangle}(\mathbf{X}, \mathbf{Y})=\varangle(\mathbf{x}, \mathbf{y})+\varepsilon d(X, Y)
$$

where the dual angle between two lines, the angle between direction vectors and the shortest distance between two lines are denoted by $\varangle(\mathbf{X}, \mathbf{Y}), \varangle(\mathbf{x}, \mathbf{y})$ and $d(X, Y)$, respectively, [3,9,10,22].

There is a one-to-one relation between lines and dual unit vectors from Study's transference principle. Then, the motion of a line in 3-dimensional real space correspond to the motion of a point on DUS, [22].

2.2.1. Dual vector representation of a ruled surface. Let $u \in \mathbb{R}$ be arbitrary parameter. The moving point $\mathbf{X}(u)=$ $\mathbf{x}(u)+\varepsilon \overline{\mathbf{x}}(u)$ on DUS demonstrate a ruled surface in 3-dimensional real space while draw a curve on DUS. If we convert the dual representation to the real one we get, $[3,8,22]$

$$
\mathbf{r}(u, v)=\mathbf{a}(u)+\lambda \mathbf{x}(u), \lambda \in \mathbb{R} .
$$

2.2.2. Dual vector representation of a line congruence. In a similar way, we can determine the dual vectorial form of the line congruence as two-parameter motion of a point on DUS

$$
\mathbf{X}(u, v)=\mathbf{x}(u, v)+\varepsilon \overline{\mathbf{x}}(u, v) .
$$

One can convert the dual representation to get the real one, $[3,8]$

$$
\mathbf{k}(u, v, w)=\mathbf{a}(u, v)+w \mathbf{x}(u, v), w \in \mathbb{R} .
$$

\subsection{Surfaces of the line congruence}

Let's look at the inner metric of the line congruence described by equation (2.3) to determine its ruled surfaces. Let take dual arc element of DUS

$$
\begin{array}{r}
d \mathbf{X}^{2}=\left[\left(\mathbf{x}_{u} d u+\mathbf{x}_{v} d v\right)+\varepsilon\left(\overline{\mathbf{x}}_{u} d u+\overline{\mathbf{x}}_{v} d v\right)\right]^{2} \\
=E d u^{2}+2 F d u d v+G d v^{2}
\end{array}
$$

then writing

$$
E=e+\varepsilon \bar{e}, F=f+\varepsilon \bar{f}, G=g+\varepsilon \bar{g}
$$

we can get

$$
\begin{array}{r}
e=<\mathbf{x}_{u}, \mathbf{x}_{u}>, \bar{e}=2<\mathbf{x}_{u}, \overline{\mathbf{x}}_{u}>, \\
f=<\mathbf{x}_{u}, \mathbf{x}_{v}>, \bar{f}=<\mathbf{x}_{u}, \overline{\mathbf{x}}_{v}>+<\mathbf{x}_{v}, \overline{\mathbf{x}}_{u}>, \\
g=<\mathbf{x}_{v}, \mathbf{x}_{v}>, \bar{g}=2<\mathbf{x}_{v}, \overline{\mathbf{x}}_{v}>
\end{array}
$$

so, first and second fundamental forms and dual arc element can be written as follows, [3]

$$
\begin{array}{r}
{[I]=e d u^{2}+2 f d u d v+g d v^{2},} \\
{[I I]=\bar{e} d u^{2}+2 \bar{f} d u d v+\bar{g} d v^{2},} \\
d \mathbf{X}^{2}=[I]+\varepsilon[I I] .
\end{array}
$$

Definition 2.2. (Distribution parameter) The ratio of the shortest distance to the angle between two infinitesimal close generator line of the ruled surfaces belong to the line congruence is called distribution parameter and is defined by mathematically

$$
\frac{1}{\delta}=\frac{d \bar{\varphi}}{d \varphi}=\frac{<d \mathbf{x}, d \overline{\mathbf{x}}>}{<d \mathbf{x}, d \mathbf{x}>}=\frac{1}{2} \frac{\bar{e} d u^{2}+2 \bar{f} d u d v+\bar{g} d v^{2}}{e d u^{2}+2 f d u d v+g d v^{2}}
$$

From equation (2.5) we can write

$$
\begin{aligned}
& (2 e-\bar{e} \delta) d u^{2}+(2 f-\bar{f} \delta) d u d v=0, \\
& (2 f-\bar{f} \delta) d u d v-(2 g-\bar{g} \delta) d v^{2}=0 .
\end{aligned}
$$


Then we have the determinant, [43]

$$
\left|\begin{array}{ll}
(e d u+f d v) & (\bar{e} d u+\bar{f} d v) \\
(f d u+g d v) & (\bar{f} d u+\bar{g} d v)
\end{array}\right|=0 .
$$

(See for more calculations [3]).

Definition 2.3. In a similar way of the theory of surfaces, the directions which providing the differential equation (2.6) are called principle surfaces of the line congruence, [3].

Definition 2.4. If the value of the distribution parameter, which is undefined, is set to infinity, then there is a special type of ruled surfaces: developable surfaces. These surfaces can be mapped isometrically into the Euclidean plane in the sense that arc lengths are preserved. For developable surfaces of the line congruences, we equate the equation (2.5) to the zero identically, [3];

$$
[I I]=\bar{e} d u^{2}+2 \bar{f} d u d v+\bar{g} d v^{2}=0 .
$$

Definition 2.5. We know that two-parameter motion of a line in 3-dimensional real space draws a line congruence. So, if we choose one of the two parameters constant, then line draws a ruled surface just as the other parameter stay constant. Then, the line of the congruence has two striction points on the each line. During two-parameter motion, these points form two surfaces:focal surfaces. All the lines of the line congruence are tangent to these surfaces at the focal points. The equations of focal surfaces are given by, $[3,11,19]$

$$
S_{1}=\mathbf{x} \times \overline{\mathbf{x}}+\frac{<\mathbf{x}_{v}, \overline{\mathbf{x}}_{u}>}{\left[\mathbf{x}, \mathbf{x}_{u}, \mathbf{x}_{v}\right]} \mathbf{x}, \quad S_{2}=\mathbf{x} \times \overline{\mathbf{x}}-\frac{<\mathbf{x}_{u}, \overline{\mathbf{x}}_{v}>}{\left[\mathbf{x}, \mathbf{x}_{u}, \mathbf{x}_{v}\right]} \mathbf{x} .
$$

Definition 2.6. The middle point of the two striction point on the main line is called central point and the equation of the locus of these points is given by

$$
\mathbf{M}(u, v)=\frac{1}{2}\left(\mathbf{S}_{1}+\mathbf{S}_{2}\right) .
$$

The equation (2.9) shows the central surface of the line congruence for all $u, v$ parameters, $[3,19]$.

\section{Methodology}

In this section, we introduce a line congruence from a two-dual parameter vector function. We previously described the function

$$
f(u+\varepsilon v)=f(u)+\varepsilon v f^{\prime}(u)
$$

where $u, v \in \mathbb{R}, f: I \subset \mathbb{D} \rightarrow \mathbb{D}$. These functions are called synectic functions, [3,22].

Similarly, we can describe one-dual parameter vector function

$$
\mathbf{X}(u+\varepsilon v)=\mathbf{X}(u)+\varepsilon v \mathbf{X}^{\prime}(u), \quad\|\mathbf{X}\|=1, \quad u, v \in \mathbb{R} .
$$

This type congruences are called by one-parameter synectic congruences, [3,22].

On the other hand, DUS can be parametrized by

$$
\mathbf{X}(\hat{u}, \hat{v})=(c(\hat{u}) s(\hat{v}), s(\hat{u}) s(\hat{v}), c(\hat{v}))
$$

since dual-valued trigonometric functions have same identities with real ones, where $\hat{u}=u+\varepsilon \bar{u}, \hat{v}=v+$ $\varepsilon \bar{v}, u, v, \bar{u}, \bar{v} \in \mathbb{R}$. Taylor expansion of dual parameter functions provides the equation (3.1) to become;

$$
\begin{aligned}
\mathbf{X}(\hat{u}, \hat{v}) & =\mathbf{X}(u, v)+\varepsilon\left[\bar{u} \cdot \mathbf{X}_{u}(u, v)+\bar{v} \cdot \mathbf{X}_{v}(u, v)\right] \\
& =(c(u) s(v), s(u) s(v), c(v))+\varepsilon[\bar{u} \cdot(-s(u) s(v), c(u) s(v), 0)+\bar{v} \cdot(c(u) c(v), s(u) c(v),-s(v))] .
\end{aligned}
$$

Similarly, this type system of congruences is called by two-dual parameter synectic congruences by Study. The equation (3.2) shows the four-parameter motion of a line in space and these parameters are freely variable and can be interconnected as desired. For the most general form of a line congruence in this line space, we assume the functions $\bar{u}=\bar{u}(u, v)$ and $\bar{v}=\bar{v}(u, v)$ and then the equation (3.2) becomes

$$
\mathbf{X}(\hat{u}, \hat{v})=\mathbf{X}(u, v)+\varepsilon \overline{\mathbf{X}}(u, v) .
$$


The equation (3.3) describes a two-real parameter motion on DUS. Other correlations between the parameters are not more general than this correlation.

Example 1. Let $\bar{u}(u, v)=u-v$ and $\bar{v}(u, v)=u+v$. Then the equation (3.3) becomes

$$
\mathbf{X}(\hat{u}, \hat{v})=(c(u) s(v), s(u) s(v), c(v))+\varepsilon[(u-v)(-s(u) s(v), c(u) s(v), 0)+(u+v)(c(u) c(v), s(u) c(v),-s(v))] .
$$

\section{Results}

All of the results were obtained from the equation (3.3) in this section.

If we put the equation (3.3) into the equation (2.4) then coefficients of first and second fundamental form become

$$
\begin{aligned}
& e=\sin ^{2} v, f=0, g=1 \\
& \bar{e}=2 \sin v\left(\frac{\partial \bar{u}}{\partial u} \sin v+\bar{v} \cos v\right), \bar{f}=\left(\frac{\partial \bar{u}}{\partial v} \sin ^{2} v+\frac{\partial \bar{v}}{\partial u}\right), \bar{g}=2 \frac{\partial \bar{v}}{\partial v}
\end{aligned}
$$

Therefore we can rewrite the distribution parameter

$$
\frac{1}{\delta}=\frac{\sin v\left(\frac{\partial \bar{u}}{\partial u} \sin v+\bar{v} \cos v\right) d u^{2}+\left(\frac{\partial \bar{u}}{\partial v} \sin ^{2} v+\frac{\partial \bar{v}}{\partial u}\right) d u d v+\frac{\partial \bar{v}}{\partial v} d v^{2}}{\sin ^{2} v d u^{2}+d v^{2}} .
$$

\subsection{Principal surfaces}

For principal surfaces, the determinate (2.6) becomes in terms of coordinate functions

$$
\left|\begin{array}{cc}
\sin ^{2} v d u & \left(2 \sin v\left(\frac{\partial \bar{u}}{\partial u} \sin v+\bar{v} \cos v\right) d u+\left(\frac{\partial \bar{u}}{\partial v} \sin ^{2} v+\frac{\partial \bar{v}}{\partial u}\right) d v\right. \\
d v & \left(\frac{\partial \bar{u}}{\partial v} \sin ^{2} v+\frac{\partial \bar{v}}{\partial u}\right) d u+2 \frac{\partial \bar{v}}{\partial v} d v
\end{array}\right|=0 .
$$

If we expand the determinate (4.3) and let $d u / d v=\zeta$, we can write;

$$
\left(\frac{\partial \bar{u}}{\partial v} \sin ^{2} v+\frac{\partial \bar{v}}{\partial u}\right) \sin ^{2} v \zeta^{2}+2 \sin ^{2} v \zeta\left(\frac{\partial \bar{u}}{\partial u}-\frac{\partial \bar{v}}{\partial v}+\bar{v} \cot v\right)+\frac{\partial \bar{u}}{\partial v} \sin ^{2} v+\frac{\partial \bar{v}}{\partial u}=0 .
$$

Equating the coefficients of the equation (4.4) to zero identically, we can write;

$$
\frac{\partial \bar{u}}{\partial v} \sin ^{2} v+\frac{\partial \bar{v}}{\partial u}=0, \frac{\partial \bar{u}}{\partial u}-\frac{\partial \bar{v}}{\partial v}+\bar{v} \cot v=0
$$

Then, we find the partially differential equations of principal surfaces of the line congruence in terms of coordinate functions.

Example 2. Let us consider the line congruence in the previous example. We know that $\bar{u}(u, v)=u-v$ and $\bar{v}(u, v)=u+v$. So we can find following differential equations

$$
\frac{\partial \bar{u}}{\partial v}=-1, \frac{\partial \bar{u}}{\partial u}=1, \frac{\partial \bar{v}}{\partial v}=1, \frac{\partial \bar{v}}{\partial u}=1 .
$$

Putting these values into the equation (4.5) we find

$$
\sin ^{2} v=1,(u+v) \cot v=0 \Rightarrow v=\frac{\pi}{2},-\frac{\pi}{2} .
$$

Then the dual vector equations of principal surfaces are (see Fig.1)

$$
\Xi_{1}(u)=(\cos u, \sin u, 0)+\varepsilon\left[\left(u-\frac{\pi}{2}\right)(-\sin u, \cos u, 0)+\left(u+\frac{\pi}{2}\right)(0,0,-1)\right]
$$

and

$$
\Xi_{2}(u)=(-\cos u,-\operatorname{sinu}, 0)+\varepsilon\left[\left(u+\frac{\pi}{2}\right)(\sin u,-\cos u, 0)+\left(u-\frac{\pi}{2}\right)(0,0,1)\right] .
$$




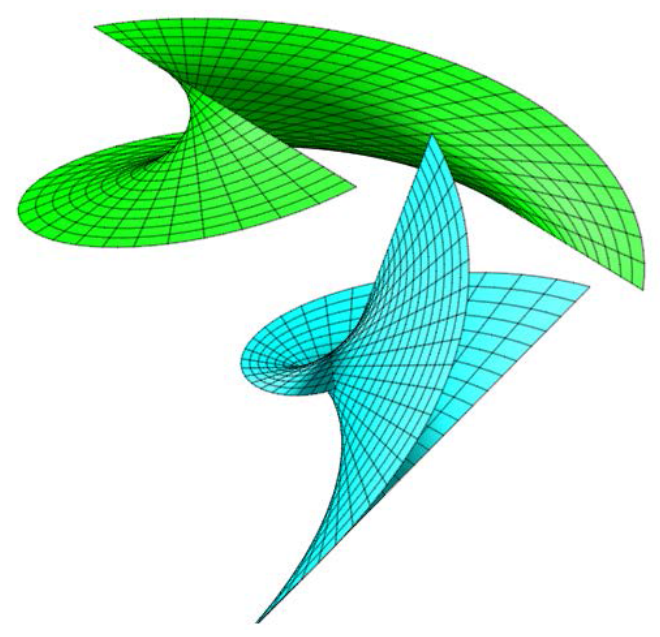

Figure 1. Principal surfaces

\subsection{Developable congruence surfaces}

For the developable surfaces of the line congruence, we put the equation (3.3) into the equation (2.7) and then find

$$
[I I]=\sin v\left(\frac{\partial \bar{u}}{\partial u} \sin v+\bar{v} \cos v\right) d u^{2}+\left(\frac{\partial \bar{u}}{\partial v} \sin ^{2} v+\frac{\partial \bar{v}}{\partial u}\right) d u d v+\frac{\partial \bar{v}}{\partial v} d v^{2}=0
$$

or equivalently

$$
\bar{e}=2 \sin v\left(\frac{\partial \bar{u}}{\partial u} \sin v+\bar{v} \cos v\right)=0, \bar{f}=\left(\frac{\partial \bar{u}}{\partial v} \sin ^{2} v+\frac{\partial \bar{v}}{\partial u}\right)=0, \bar{g}=2 \frac{\partial \bar{v}}{\partial v}=0 .
$$

From the solutions of the above partial differential equations we get

$$
\bar{v}=c_{1} \cos u+c_{2} \sin u, \bar{u}=\cot v\left(c_{2} \cos u-c_{1} \sin u\right)
$$

where $c_{1}$ and $c_{2}$ are integral constants. Therefore, the dual vector equation of the developable congruence surfaces is

$$
\begin{aligned}
\mathbf{X}(u, v)= & (c(u) s(v), s(u) s(v), c(v))+ \\
& \varepsilon\left[\cot (v)\left(c_{2} c(u)-c_{1} s(u)\right) \cdot(-s(u) s(v), c(u) s(v), 0)+\left(c_{1} c(u)+c_{2} s(u)\right) \cdot(c(u) c(v), s(u) c(v),-s(v))\right] .
\end{aligned}
$$

4.3. Focal surfaces and central surface

From (3.3) and (2.8), the equations of focal surfaces are represented by

$$
\begin{gathered}
\mathbf{S}_{1}=\left(-\bar{v} \sin u-\frac{\partial \bar{v}}{\partial u} \cos u, \bar{v} \cos u-\frac{\partial \bar{v}}{\partial u} \sin u, \bar{u}-\frac{\partial \bar{v}}{\partial u} \operatorname{cotu}\right) \\
\mathbf{S}_{2}=\left(-\bar{v} \sin u+\frac{\partial \bar{u}}{\partial v} \operatorname{cosusin}^{2} v, \bar{v} \cos u+\frac{\partial \bar{u}}{\partial v} \operatorname{sinusin}^{2} v, \bar{u}+\frac{\partial \bar{u}}{\partial v} \cos v \sin v\right)
\end{gathered}
$$

Therefore we can write the equation of central surface in terms of coordinate functions like this

$\mathbf{M}(u, v)=\left(-\bar{v} s(u)-\frac{1}{2} \frac{\partial \bar{v}}{\partial u} c(u)+\frac{1}{2} \frac{\partial \bar{u}}{\partial v} c(u) s^{2}(v), \bar{v} c(u)-\frac{1}{2} \frac{\partial \bar{v}}{\partial u} s(u)+\frac{1}{2} \frac{\partial \bar{u}}{\partial v} s(u) s^{2}(v), \bar{u}+\frac{1}{2} \frac{\partial \bar{u}}{\partial v} c(v) s(v)+\frac{1}{2} \frac{\partial \bar{v}}{\partial u} c(v)\right)$.

\section{Conclusion}

In this paper, we examined that the differential geometric properties of the line congruences. Therefore, equations of the surfaces of a line congruence have been obtained in terms of coordinate functions. Especially, it is important that getting an explicit equation which belongs to the developable surface of the line congruence. 
So, we have found general solutions for determining the surfaces of the line congruence. For the future studies, it is possible to look at the ruled surfaces of this study. In addition, other types of congruence can be researched. Specifically, the type of focal surfaces can be determined and the link between them can be investigated. Of course, the motion of the spacelike and timelike vectors can be studied with the Minkowski space-time geometry in a motion of the type in this study.

\section{References}

[1] Abdel-Baky, R.A., Al-Bokhary, A.J., A new approach for describing instantaneous line congruence, Archivum Mathematicum, (2008), pp. 223-236.

[2] Ball, R.S., A Treatise on the Theory of Screws, Cambridge University Press, 1900 (reprinted 1999).

[3] Blaschke, W., Vorlesungen über differential geometrie I, Springer-Verlag, 1945, Berlin.

[4] Bottema, O., Roth, B., Theoretical Kinematics, Dover Publications, 1990.

[5] Clifford, W.K., Preliminary sketch of bi-quaternions, Proceedings of the Royal Society of London, (1873), pp. 381-395.

[6] Dimentberg, F.M., The Screw Calculus and Its Applications in Mechanics, Foreign Technology Division translation, FTD-HT-1632-67, 1965.

[7] Eisenhart, L.P., A Treatise in Differential Geometry of Curves and Surfaces, New York, Ginn Camp., 1969.

[8] Guggenheimer, H.W., Differential geometry, Dover Publications, Inc. New York, 1977.

[9] Gürsoy, O. J Geom (1990) 39: 80. https://doi.org/10.1007/BF01222141.

[10] Gürsoy, O., Some results on closed ruled surfaces and closed space curves, Mech. Mach. Theory, (1992), pp.323-330.

[11] Hlavaty, V., Differential line geometry, Groningen, P.Noordhoff Ltd. X, 1953.

[12] Hoschek, J., Liniengeometrie, B.I. Hochschultaschenbuch, Mannheim, 1971.

[13] Hunt, K.H., Kinematic Geometry of Mechanisms, Oxford Press, 1978.

[14] Jüttler, B., Rittenschober, K., Using line congruences for parametrizing special algebraic surfaces, The Mathematics of Surfaces X, Lecture Notes in Computer Science, vol.2768, Springer-Berlin, 2003, pp. 223-243.

[15] Köse, Ö., A method of the determination of a developable ruled surface, Mech. Mach. Theory, (1999), pp. 1187-1193.

[16] Larochelle, P.M., Vance, J.M., Kihonge, J.N., Interactive visualization of line congruences for spatial mechanism design, Journal of Computing and Information Science in Engineering, (2002), pp. 208-215.

[17] Odehnal, B., On rational isotropic congruences of lines, Journal of Geometry, (2004), pp. 126-138.

[18] Plücker, J., On a new geometry of space, Proceedings of the Royal Society of London, (1865), pp. 53-58.

[19] Pottmann, H., Wallner, J., Computational line geometry, Mathematics and Visualization, Springer-Verlag Berlin Heidelberg, 2001.

[20] Selig, J.M., Geometrical Methods in Robotics, Springer, New York, 1994.

[21] Shepherd, M.D., Line congruences as surfaces in the space of lines, Differential Geometry and its Applications, (1999), pp. 1-26.

[22] Study, E., Geometry der dynamen, Leipzip, 1903.

[23] Veldkamp, G.R., On the use of dual numbers vectors and matrices in instantaneous spatial kinematics, Mech. Mach. Theory, (1976), pp. $141-156$.

\section{Affiliations}

FERHAT TAŞ

ADDRESS: Department of Mathematics, Faculty of Science, Istanbul University, 34134, Istanbul, Turkey

E-MAIL: tasf@istanbul.edu.tr

ORCID ID : orcid.org/0000-0001-5903-2881

\section{OSMAN GÜRSOY}

AdDRESs: The Department of Mathematics and Science Education, Faculty of Education, Marmara Eğitim Köyü, 34857, Maltepe, Istanbul, Turkey.

E-MAIL: osmangursoy@maltepe.edu.tr

ORCID ID : orcid.org/0000-0002-6391-6214 